\title{
Evaluation of the usefulness of a computer- based learning program to support student learning in pharmacology
}

\author{
S. Brain,* D. G. Dewhurst** and A. D.Williams*** \\ *Biomedical Sciences Division, King's College; **Learning Technology Section, Faculty \\ Group of Medicine \& Veterinary Medicine, University of Edinburgh; *** School of \\ Health Sciences, Leeds Metropolitan University. Email: d.dewhurst@ed.ac.uk
}

This study aims to evaluate the effectiveness of a computer-based teaching program in supporting and enhancing traditional teaching methods. The program covers the pharmacology of inflammation and has been evaluated with a group of second-year medical students at a UK university. The study assessed subject-specific knowledge using a pre- and post-test and surveyed, by questionnaire, students' perceptions of the usefulness of the program to support learning before and after use. The use of computers for learning amongst this cohort of students was widespread. The results demonstrated an increase in students' knowledge of the pharmacology of inflammation, coupled with a positive attitude towards the CBL program they had used and the advantages that this mode of study may provide in enabling students to manage their own learning. However, students did not feel that the program could substitute for traditional teaching (lectures).

\section{Introduction}

The use of computers in undergraduate education is now widespread and computer-based learning (CBL) has made a significant impact in teaching, particularly over the last decade. This is likely to increase substantially as some $£ 100$ million has been made available to stimulate software development in the UK through various initiatives, such as the EEC COMETT program and the UK Higher Education Funding Councils' Teaching and Learning Technology Programme (TLTP).

CBL has a number of perceived advantages to both staff and students: it can promote active, rather than passive, learning; it can give students control over when and where they learn and the pace of their learning; it may promote better understanding by using features which both enhance the clarity and quality of presentation of material and stimulate student interaction; it 
can potentially save staff time and resources. It especially lends itself to the teaching of medicine, which as Waterfield and Furber (1992) emphasize is a highly visual subject.

At King's College, London, CBL is increasingly used as a teaching and learning tool in the undergraduate medical curriculum. This is in response to the perceived need that medical graduates need to be both familiar with and have a threshold competence in information technology and computing skills (Clack, 1994; GMC, 1993). Furthermore, there is now an abundance of CBL resources available for supporting teaching and learning in the medical sciences, particularly material produced by TLTP projects in medicine-related subjects.

In the biomedical sciences a number of studies have assessed the effectiveness of different forms of CBL. This includes studies on computer-based simulations of laboratory practicals (Clarke, 1987; Brown et al., 1988; Modell, 1989, 1992a; Fawver et al., 1990; Guy and Frisby, 1992; Coleman et al., 1994; Dewhurst et al., 1994; Leathard and Dewhurst, 1995; McAteer et $a l ., 1996$ ) and computer-based tutorials (Anderson-Harper et al., 1988; Fincher $e$ t al., 1988; Feldman et al., 1989; Garrett et al., 1990; Hollingsworth and Foster, 1991; McCurrie and Huckbody, 1992; Modell, 1992b; Hutchins et al., 1995; Inglis et al., 1995; Gunn and Maxwell 1996). In the main, such studies conclude that CBL is at least as effective (in terms of knowledge acquisition or meeting assessment criteria) as traditional methods of teaching, although its use may result in different learning objectives being achieved - for example, computer simulations of laboratory-based experiments cannot teach practical laboratory skills.

Many teachers are unwilling to use CBL packages, particularly those which are developed by others outside their own university department, and studies which evaluate their use may be helpful in breaking down barriers to new methods of teaching and learning. Here we describe a study that assesses the effectiveness of a CBL program on inflammation in supporting the teaching of undergraduate medical students.

\section{The computer program}

The CBL program used in the study was developed by the authors as part of a TLTP project (Pharma-CAL-ogy). It is intended as an introductory learning package on the pharmacology and pathophysiology of inflammation, and is aimed primarily at undergraduate students of pharmacology, medicine, dentistry and biological sciences.

The anticipated learning outcomes are that, on completion of the package, students should be able to:

- describe the timescale and the cardinal signs of the inflammatory response to tissue injury;

- list the various chemical mediators involved and describe and explain their local and systemic effects;

- explain the molecular mechanisms of cell accumulation;

- describe the major processes involved in tissue repair;

- describe and explain the pharmacological actions of the major classes of anti-inflammatory drugs. 
The package covers the following subjects:

- the main elements of inflammation, major cardinal signs and the timescale of the various processes involved in the inflammatory response;

- the microcirculation;

- inflammatory mediators - the synthesis, site and mechanism of actions of histamine, bradykinin, nitric oxide, eicosanoids, neuropeptides and cytokines;

- cell accumulation - molecular mechanisms involved in neutrophil accumulation at the site of injury, adherence, margination and extravasation;

- mechanisms of tissue repair and healing;

- the pharmacology (name, structure, mechanism of action, and toxicity) of the main drugs: non-steroidal anti-inflammatory drugs (NSAIDs); steroidal anti-inflammatory drugs (SAIDs); disease modifying anti-arthritic drugs (DMARDs).

The program is highly interactive and makes extensive use of colour graphics (there is a graphic on most screens), animations (particularly to illustrate dynamic mechanisms like neutrophil accumulation, action of mediators and anti-inflammatory agents at cell receptors), a hotword facility (to give additional information on certain topics), a glossary (to define terms which may be unfamiliar to students) and features to promote interactivity, e.g. 'drag and drop' exercises, self-assessment questions (MCQs and true/false with feedback). The content and approach is described in more detail elsewhere (Dewhurst et al., 1996).

\section{The summative evaluation}

\section{Method}

The study was conducted with a single cohort $(n=78)$ of second-year medical students at King's College, London. The study assessed student knowledge of the subject area covered by the CBL program and students' attitudes to using CBL compared with traditional teaching methods.

Students attended a series of lectures covering five topics in pharmacology. One topic, inflammation, was delivered via four one-hour lectures and covered anti-arrhythmic drugs, general anaesthetics, muscle relaxants, opioids and inflammation. Two months later the CBL program was used as part of a series of timetabled three-hour sessions designed to support these lectures. Small groups of students (20-25) rotated around each of the support sessions and in the sixth week, when the series was completed, they attended debriefing tutorials. The format and approach of the support sessions varied. For example, the anti-arrhythmic support session involved a trip to theatre and had much more obvious clinical relevance. The CBL package was used to provide an additional and different style of learning resource to the lectures, and staff running the course felt that it was particularly useful in the area of therapeutics and antiinflammatory drugs, which were not covered adequately in the lectures.

\section{Assessment of student knowledge}

A multiple choice question (MCQ) paper was used as a pre- and post-test to assess students' knowledge of inflammation. The paper contained 22 questions, each consisting of a number of items with which the students were required to indicate their agreement or disagreement. Students were instructed not to attempt any items they felt particularly unsure about. 
The pre-test paper was administered during the support session before the students used the CBL program. The post-test paper, depending on the cohort of students, was administered during the debriefing tutorial. When they had completed the CBL session students were informed that they could continue to use the program for independent study.

\section{Assessment of student attitude}

An attitudinal questionnaire was devised which consisted of questions selected to provide information on three attitude scales (delivery, knowledge, and time and place). This was then used as a pre- and post-measure of attitude towards CBL in relation to traditional lectures. Students completed the questionnaire at the same time as they completed the knowledge test outlined above. The attitude scales used consisted of additive Likert items (Likert, 1932). The delivery scale consists of five items (questions) and is interpreted as measuring a positive attitude to 'whether CBL can replace lectures as an effective delivery medium'. Similarly, the knowledge scale consists of four items: positive attitude to 'as much knowledge can be gained from CBL programs as from lectures' and the time and place scale consists of three items: 'CBL allows students to determine time, place and pace of learning'. The construct validity of the scales was assessed using principal component factor analysis and the reliabilities quoted are Cronbach's alpha coefficient. Each item on a scale has five points scored from +2 (strongly agree) through 0 (undecided) to -2 (strongly disagree). The item responses for a particular scale are summed to give that scale's total response. The reliability of the scales were as follows: delivery (pre-test: 0.80 ( $n=149)$; post-test: $0.78(\mathrm{n}=133))$; knowledge (pre-test: $0.70(\mathrm{n}=149)$; post-test: 0.67 $(n=132)) ;$ time and place (pre-test: $0.54(n=149)$; post-test: $0.74(n=132))$. Here $n$ corresponds to the number of responses on a particular scale using data from this study and a separate study (carried out at Leeds Metropolitan University). The pre-test reliability of the time and place scale is low (which could be due to the small number (three) of items in the scale); however, the posttest reliability of this scale was more acceptable and so the scale was retained for the purposes of this study.

An additional scale (ease of use) was constructed, for use as a post-test measure, to assess the students' impressions of the specific CBL program used in this study. This scale had six items covering 'The CBL program was easy to use' and had a reliability of $0.74(n=133)$. The questionnaire also contained a question asking for comments on general factors such as ease of use, program design and content.

\section{Results}

\section{Student knowledge}

The results of the pre-and post- knowledge tests are presented in Figure 1. A substantial increase in knowledge ( $p<0.001$, one-tailed paired $t$-test) was seen in the post-test scores (mean \pm sd: $83.8 \pm 10.4$; min: 46 ; max: 96), when compared with the pre-test scores (mean \pm sd: 58.0 \pm 14.4 ; $\min : 22$; $\max : 82$ ).

\section{Student attitude}

There were 78 responses on each scale in the pre-test attitude questionnaire and 70 responses on each scale in the post-test questionnaire. The pre-test attitude on the delivery scale was negative $(\mathrm{p}<0.001$, Wilcoxon signed-rank test). Pre-test attitude on the knowledge scale was not significantly different from the zero (undecided) point of the scale $(\mathrm{p}=0.43)$. There was a significant positive attitude on the time and place scale $(\mathrm{p}<0.001)$. Analysis of the post-test results showed no change in attitude on any of the scales used (Table 1). However, the p-value 


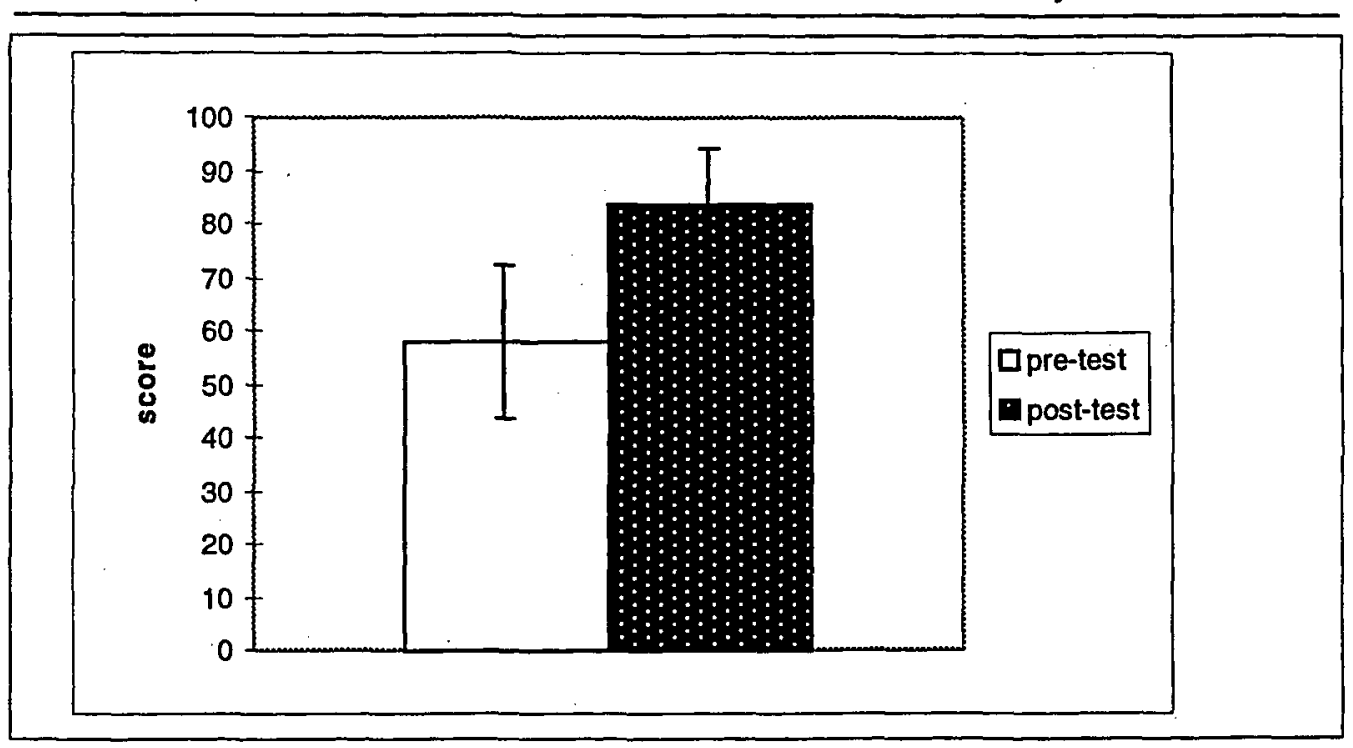

Figure 1: Pre- and post-test mean knowledge.

A substantial increase in mean knowledge $(p<0.001$, one-tailed paired $t$-test) was seen on the posttest. Error bars indicate standard deviation. Maximum possible score $=102$.

for the knowledge scale was low $(\mathrm{p}=0.11)$ and an analysis of post-test scores on this scale against a median of zero gave a significant result ( $\mathrm{p}=0.02$, Wilcoxon signed-rank test).

The post-test ease of use scale showed a positive attitude (Table 1). The majority of students ( $>80$ per cent) found the program easy to use and liked many aspects of the program including:

- presentation of textual information;

- use of subsections;

- level of detail in textual information;

- opportunity to gain more information by highlighting text/graphics;

- flow diagrams;

- graphics;

- question and answer sections.

Most of the negative comments related to the time taken to use the program, as is illustrated in the quote 'the program was boring, too lengthy and overloaded with information'.

Students generally felt that they had extended both the range (81 per cent) and depth (68 per cent) of their knowledge about inflammation, but were unclear ( 62 per cent) about the clinical and therapeutic aspects of the pharmacology of inflammation. 


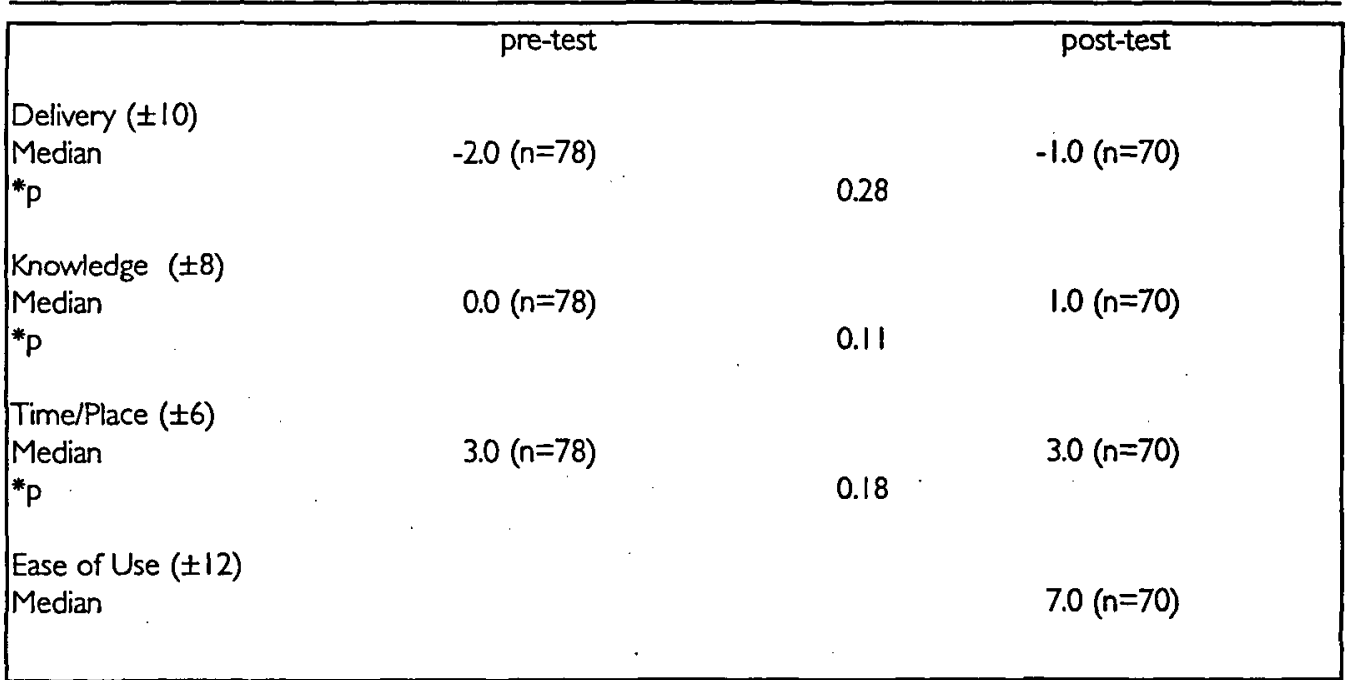

Table 1: Pre- and post-test attitudes towards CBL and post-test rating of the ease of use of the program.

*p-values from Wilcoxon signed-rank tests (two-tailed) examining differences in median attitude score before and ofter using CBL.

Pre-test attitude on the Delivery scale was slightly negative ( $p<0.001$, Wilcoxon signed-rank test). No change in attitude was seen in the post-test $(p=0.28)$. Pre-test attitude on the Knowledge scale was not significantly different from the zero point (undecided; $p=0.43$ ). There was no change in attitude on the Knowledge scale although the $p$-value was low $(p=0.11)$ and an analysis of post-test scores on this scale against a median of zero gove a significant result ( $p=0.02$. Wikcoxon signed-rank test). There was a significant positive attitude on the Time and Place scale $(p<0.001)$ for both pre- and post-tests. The Ease of Use scale used only as a post-test measure showed a positive attitude.

\section{Assessment of students' prior experience}

All students in the study were familiar with using computers, with a significant number (62 per cent) of students who used computers both inside and outside college. However, only a minority (26 per cent) claimed to use them frequently. With the exception of one student, who had not used a CBL program before, the majority (78 per cent) had used CBL programs at least three times prior to the inflammation CBL.

\section{Discussion}

This study relates to one cohort of students at one university using one specific CBL package in one prescribed way and therefore it is impossible to generalize any conclusions. However, there are three major findings from the study. First, it is evident that the use of computers is widespread amongst medical students at King's and that many students are using computers for learning. Second, using the CBL program as a support to lectures does reinforce knowledge of the topic - a significant increase in knowledge was seen after the CBL session on an already high pre-test knowledge base. (In this study no attempt was made to compare learning through CBL with learning through lectures.) Third, students had a slightly negative opinion of CBL as 
a method of delivery compared to lectures both before and after they used the CBL program, although they did recognize the potential benefits of CBL in terms of study management.

The increase in knowledge was perhaps predictable as students were tested shortly (one to six weeks) after they had used the CBL program. We attribute the comparatively high pre-test scores to students having previously attended lectures on the topic two months earlier. It would be interesting to assess retained knowledge, perhaps one year later, though it is impossible to control what additional subject-specific learning students engage in during that period. The results do indicate that a relatively short $\mathrm{CBL}$ session would be a useful way for students to revise prior to exams and may be an effective means of introducing students to this form of learning.

The attitude scales described here have also been used (by the authors) in a similar study (topic area: cardiovascular physiology) with another group of students in a different university. The results of this study are similar and suggest that while students do not feel that CBL provides as enjoyable an environment for learning as traditional lectures they are willing to accept that they can in fact learn as much from either approach. What we may be able to conclude from this is that students appear to be most comfortable with a learning environment in which CBL is integrated into a course in which a variety of delivery methods are used.

In this study the negative attitude to CBL did not seem to be due to students feeling that the particular CBL program they used was poor. The ease of use scale reflected that opinion was generally favourable and that most students felt they had extended the range and depth of their knowledge base. It should be noted that a significant proportion ( 48 per cent) of the students did not feel that they had a clear knowledge of the therapeutics of inflammation after using the program. This was an unexpected response as the program includes a detailed section on this topic. One explanation is that the students did not have time in the three-hour session to go through this section (many did indeed report that the program was over-long), alternatively it could be that the mechanistic approach taken by the authors in this section was insufficiently clinical. In general the package is content-rich and probably contains far too much information for one three-hour session. Students tended to try to work through the package from start to finish rather than select areas which cover parts of the course they feel less confident about. To address this it may be appropriate for tutors to direct students to specific sections of the package.

In this instance it may be possible to rewrite this section to accommodate some of the students' comments, but it does highlight a potential problem for other educators who may wish to make use of commercial software not developed in-house. Many might reject a particular program if part of it is unsuitable, even if the rest of the program meets their learning objectives. For developers it suggests that shorter programs covering small topics, which may then be assembled by the user into a larger program, may be more useful.

In the future the program will be used at King's as an integral part of teaching for medical students and also for students on a range of non-clinical/biochemical courses.

\section{References}

Anderson-Harper, H. M., Mason, H. L. and Popovich, N. G. (1988), 'Developing, implementing and evaluating microcomputer instruction in a non-prescription drug course', American Journal of Pharmaceutical Education, 52, 259-66. 
Brown, G. J., Collins, G. G. S., Dewhurst, D. G. and Hughes, I. E. (1988), 'Computer simulations in teaching neuromuscular pharmacology - time for a change from traditional methods?', Alternatives to Laboratory Animals, 16, 163-74.

Clack, G. B. (1994), 'Medical graduates evaluate the effectiveness of their education', Medical Education, 28, 418-31.

Clarke, K. A. (1987), 'The use of microcomputer simulations in undergraduate neurophysiology experiments', Alternatives to Laboratory Animals, 14, 134-40.

Dewhurst, D. G., Hardcastle, J., Hardcastle, P. T. and Stuart, E. (1994), 'Comparison of a computer simulation program with a traditional laboratory practical class for teaching the principles of intestinal absorption', Amer. J. Physiol. (Adv. Physiol. Educ.), 12, 267, S95-S104.

Dewhurst, D. G., Brain, S. D., Freeman, P. and Ullyott, R. T. (1996), 'The pathophysiology and pharmacology of inflammation - an interactive computer-based tutorial program for undergraduate students', Brit. J. Pharmac. Proc. Suppl., 118, 155P.

Fawver, A. L., Branch, C. E., Trentham, L., Robertson, B. T. and Beckett, S. D. (1990), 'A comparison of interactive videodisc instruction with live animal laboratories', Amer. J. Physiol. (Adv. Physiol. Educ.), 4, 259, S11-S14.

Feldman, R. D., Schoenwald, R. and Kane, J. (1989), 'Development of a computer-based instructional system in pharmacokinetics, efficacy in clinical pharmacology teaching for senior medical students', Journal of Clinical Pharmacology, 29, 158-61.

Fincher, R. M. E., Abdulla, A. M., Sridharan, M. R., Houghton, J. L. and Henke, J. S. (1988), 'CAL compared with weekly seminars for teaching fundamental electrocardiography to junior medical students', Southern Medical Journal, 81, 10, 1291-4.

Garrett, T. J., Selnow, G., Dobkin, J. F. and Healton, C. (1990), 'Computer-assisted instruction in AIDS infection control for physicians', Teaching and Learning in Medicine, 2, 215-18.

General Medical Council (1993), Tomorrow's Doctors.

Gunn, C. and Maxwell, L. (1996), 'CAL in human anatomy', Journal of Computer Assisted Learning, 12, 4, 205-15.

Guy, J. F. and Frisby, A. J. (1992), 'Using interactive videodiscs to teach gross anatomy to undergraduates at the Ohio State University', Acad. Med., 67, 132-3.

Hollingsworth, M. and Foster, R. W. (1991), 'Computer-assisted learning (CAL) programs in drug disposition: replacement of lectures', Brit. J. Pharmac. Proc. Suppl., 102, 216P.

Hutchins, L. F., Fulper-Smith, M., Deloney, L., Gest, T. R., Roberson, P. K. and Berry, D. H. (1995), 'Evaluation of computer-based cancer education modules at the Arkansas Cancer Research Center', Journal of Medical Education Technologies, 6, 1, 4-9.

Inglis, T., Fu, B. and Kwokchan, L. (1995) 'Teaching microbiology with hypertext: first steps towards a virtual textbook', Medical Education, 29, 6, 393-6.

Leathard, H. L. and Dewhurst, D. G. (1995) 'Comparison of the cost-effectiveness of a computer-assisted learning program with a tutored demonstration to teach intestinal motility to medical students', ALT-J, 3 (1), 118-25. 
Likert, R. A. (1932), 'A technique for the measurement of attitudes', Archives of Psychology, 140.

McAteer, E., Neil, D., Barr, N., Brown, M., Draper, S. and Henderson, F. (1996), 'Simulation software in a life sciences practical laboratory', Computers and Education, 26, 1, 101-12.

McCurrie, J. and Huckbody, K. (1992), 'Introducing computer assisted learning into physiology teaching for students of pharmacy and other health science courses', Proceedings of the CTI Centre for Medicine Conference, 23-4.

Modell, H. I. (1989), 'Can technology replace live preparations in student laboratories?', Amer. J. Physiol. (Adv. Physiol. Educ.), 256, 1, S18-S20.

Modell, H. I. (1992a), 'Developing simulations to help students learn renal physiology', Computers in Life Science Education, 9, 4, 65-77.

Modell, H. I. (1992b), 'Computer software for physiology education', Amer. J. Physiol. (Adv. Physiol. Educ.), 261, 6, S29-S33.

Waterford, M. and Furbur, S. (1992), 'Computers in teaching medicine', Computers and Education, 19, 1, 145-51. 